\title{
AN IMPROVED SYNTHETIC APERTURE RADAR IMAGE DENOISING METHOD BASED ON BLOCK-MATCHING AND 3D FILTERING
}

\author{
Xueqing $\mathrm{Liu}^{1,2}$, Xiaoping $\mathrm{Lu}^{1, *}$, Liangting $\mathrm{Ma}^{1,2}$ \\ ${ }^{1}$ Key Laboratory of Spatio-temporal Information and Ecological Restoration of Mines of Natural Resources of the People's Republic \\ of China, Henan Polytechnic University, Jiaozuo 454003, China - lxp@ hpu.edu.cn \\ ${ }^{2}$ School of Surveying and Land Information Engineering, Henan Polytechnic University, Jiaozuo 454003, China - 211804020053 \\ @ home.hpu.edu.cn, $871451582 @ q q . c o m$
}

Commission III, WGIII/3

KEY WORDS: Synthetic Aperture Radar, Speckle Noise, Denoising, Block-Matching And 3D Filtering, Block Similarity Measure

\begin{abstract}
:
Block-matching and 3D filtering (BM3D) are used to reduce the multiplicative coherent speckle noise of Synthetic Aperture Radar (SAR) images, which may lead to the loss of image details. This paper proposes an improved similarity metric BM3D algorithm. Firstly, this method analyses the coherent speckle noise model, and applies a logarithmic transformation to make the BM3D algorithm suitable for multiplicative noise. Secondly, this method based on the calculation method of Euclidean distance weights for similar image blocks, and the Pearson correlation coefficient is introduced to improve the similarity metric. The accuracy of similar image block matching is improved, which is beneficial for removing image noise and maintaining image information. The experiments in this paper compared the results of this method with Frost filtering, Kuan filtering, wavelet soft thresholding and SAR-BM3D filtering algorithms. The results were compared and analysed by subjective vision and objective indicators. The experimental results show that compared with other filtering algorithms, the proposed algorithm has better ability to reduce speckle noise and preserve edge detail information for the image.
\end{abstract}

\section{INTRODUCTION}

Synthetic Aperture Radar (SAR) images are different from optical images dominated by additive Gaussian noise, which is dominated by multiplicative noise (i.e. coherent speckle noise) (Wang et al., 2017). Coherent speckle noise will reduce the spatial resolution of SAR images and lose structure information, which interprets SAR images more complicated (Xu et al., 2000). At present, the mainstream coherent speckle suppression methods are divided into two types: speckle reduction filtering in the spatial domain and the transform domain. The typical spatial domain filtering methods include Lee filtering, Frost filtering, and Gamma-MAP filtering, etc. This type of method is easy to process and has good denoising performance, but it will cause the image to be blurred and lose the detailed information of the image. The typical transform domain filtering methods include Fourier transform, wavelet transform, multi-scale geometric filtering methods, etc. These methods can better maintain edge and detail information for the image, but the image will still be a lot of speckle noise. Both types of filtering methods are calculated based on local models, which only consider local information of the image, and do not make full use of image structure information for denoising processing (Chen, $\mathrm{Li}, 2017)$. Under this background, Buades et al. (Buades et al., 2005) proposed a non-local means (NL-Means) filtering for image additive noise and verified that the NL-Means filtering algorithm has better denoising effect than the local smoothing algorithm. In 2007, Dabov et al. (Dabov et al., 2007) uses the NL-Means filtering idea to combine the transform domain and non-local mean methods to perform non-local modelling by aggregating similar image blocks in a three-dimensional array. They proposed a BM3D algorithm for image denoising, which has both good denoising performance and subjective visual quality.
At present, the denoising of SAR images using the BM3D algorithm has become a research hotspot. In 2014, Cozzolino et al. (Cozzolino et al., 2014) proposed a fast-non-local speckle filtering method for the BM3D algorithm to calculate the similarity measure of many matching blocks. This method generates a variable size search area from the activity level of each block. The speckle statistics are used to speed up the matching probability of image blocks, which can maintain the excellent denoising performance of the BM3D algorithm and improve the operation efficiency of the algorithm. In 2015, Muralimohanbabu et al. (Muralimohanbabu et al., 2015) proposed a BM3D algorithm using a non-sampling wavelet transform, which can better remove coherent speckle noise and maintain edge features. In 2017, Chierchia et al. (Chierchia et al., 2017) used SAR-BM3D extension to process multi-temporal SAR data and proposed a multi-temporal synthetic aperture radar image removal speckle-noise algorithm based on block-matching and collaborative filtering. This algorithm can suppress speckle noise and artefacts while retaining the image fine structure and region boundaries. BM3D algorithm uses the similarity between 'blocks' to achieve high sparseness of the image through joint filtering. This method has good denoising performance and has more advantageous than other methods (Zhao et al., 2019). However, there is also a problem that when the noise reaches a certain level, the denoising performance of BM3D decreases. The main reason is that the accuracy of similar block matching will decrease sharply after the noise becomes stronger (Dabov et al., 2007). Therefore, based on the analysis of the coherent speckle noise model, this paper proposes a BM3D algorithm that improves the similarity measurement by the Pearson correlation coefficient and compares it with various filtering methods. The experimental results show that the proposed algorithm can

\footnotetext{
* Corresponding author
} 
effectively remove coherent speckle noise and better retain the edge detail information of the image.

\section{METHODS}

The BM3D algorithm can effectively suppress additive Gaussian noise and is not suitable for SAR images with multiplicative coherent speckle noise. Based on the coherent speckle noise model of the SAR image, this paper applies the logarithmic transformation to convert it to additive noise and uses the Pearson correlation coefficient to improve the similarity criterion of Euclidean distance metric in BM3D algorithm to improve image denoising performance.

\subsection{Coherent Speckle Noise Model}

SAR images are formed by coherently superimposing radar pulse echoes and compressing them in azimuth and distance. Among them, echoes of many scattering points randomly distributed within the same resolution are coherently superimposed to form coherent speckle noise (Li, Shen, 2016). Arsenault and April (Arsenault, April, 1976) proved that the coherent speckle noise can be expressed as a multiplicative noise model. It is assumed that the intensity value of the SAR image is equal to the product of the average scattering intensity value in the target resolution unit and the coherent speckle noise. It can be transformed to approximately Gaussian additive noise by the logarithmic transformation. The multiplicative noise model is:

$$
I(i)=Q(i) \cdot u(i)
$$

where $\quad I(i)=$ the intensity of the image with noise

$Q(i)=$ the intensity of the image without noise

$u(i)=$ the intensity of the coherent speckle noise

Equation (1) performs logarithmic transformation to obtain the additive noise model:

$$
\log I(i)=\log Q(i)+\log u(i)
$$

\subsection{BM3D}

BM3D algorithm is based on block matching in the threedimensional transform domain filtering. It searches for similar blocks of the image by Euclidean distance to match and constructs a three-dimensional image block matrix, and performs collaborative filtering and aggregation processing to achieve image noise reduction. Its technical process is divided into two steps: basic estimation and final estimation.

2.2.1 Basic Estimation: Grouping. Select a reference block $P$ of size $N^{2}$ in the noisy image $I$, apply the Euclidean distance to search for similar blocks according to the set search window and form a similar block matrix set $B P$, then obtain a threedimensional matrix $G(P)$ after integration.

$$
G(P)=\{Q: d(P, Q) \leq \tau\}
$$

where $\quad \tau=$ the distance threshold

$N^{2}=$ the size of the reference block

$S(P)=$ the matrix value of the reference block

$S(Q)=$ the matrix value of the matching block

$d(P, Q)=\frac{\|S(P)-S(Q)\|^{2}}{N^{2}}$, Euclidean distance

between the reference block $P$ and the matching block $Q$
Cooperative filtering. According to the three-dimensional matrix $G(P)$ that has been formed above. It is subjected to threedimensional transformation, hard threshold shrinkage, and threedimensional inverse transformation to obtain the processed image block $R(P)$.

$$
R(P)=T_{3 D}^{-1}\left(\gamma\left(T_{3 D}(G(P))\right)\right)
$$

Where $\quad T_{3 D}=$ three-dimensional transformation of the matrix $G(P)$ matrix $G(P)$

$T_{3 D}{ }^{-1}=$ three-dimensional inverse transformation of the

$x=$ the number of similar block matrix values

$\lambda_{3 D}=$ the hard threshold shrinkage parameter

$\sigma=$ the standard deviation of noise

$\gamma=\left\{\begin{array}{l}0, X \leq \lambda_{3 D} \sigma \\ x, X>\lambda_{3 D} \sigma\end{array}\right.$, the estimated value of the hard

threshold

Aggregation. After the hard threshold shrinking, each image block or pixel will get estimated value $i$, and the same pixel $i$ may appear in multiple different blocks. It is necessary to make a weighted average of the estimated value of these overlapping blocks to calculate the basic estimated value of $i$ (Shi et al., 2016).

$$
Q_{\text {basic }}(i)=\frac{\sum_{\mathrm{P}} \sum_{\mathrm{Q} \in \mathrm{B}_{P}} W_{P}^{\text {basic }} R_{P Q}}{\sum_{\mathrm{P}} \sum_{\mathrm{Q} \in \mathrm{B}_{P}} W_{P}^{\text {basic }} X_{Q}}, i \in I
$$

where $R_{P Q}=$ the estimated value of the three-dimensional transformed block containing pixel $i$ in any set $B_{P}$

$N_{P}=$ the non-zero number of matrix values of the threedimensional block after hard threshold filtering

$$
W_{P}^{\text {basic }}=\left\{\begin{array}{l}
\frac{1}{N_{P}}, N_{P} \geq 1 \\
1, N_{P}<1
\end{array}\right\} \text {, the basic estimated weight }
$$

2.2.2 Final Estimation: Grouping. We will get two threedimensional matrices. One is a three-dimensional matrix $G(P)$ formed by the noisy image, and the other is to use similar Euclidean distance to perform similar block-matching on the image blocks obtained from the basic estimation to form a new three-dimensional matrix $G^{\prime}(P)$.

Cooperative filtering. Perform three-dimensional transformation on both three-dimensional matrices, use Wiener filtering to scale the three-dimensional matrix formed by the basic estimation, and then perform a three-dimensional inverse transformation.

$$
R^{\prime}(P)=T_{3 D}^{-1}\left(W_{P}\left(T_{3 D}\left(G^{\prime}(P)\right)\right)\right)
$$

where $\quad W_{P}=$ the coefficient of Wiener filter

Aggregation. In the same way as the basic estimation, these blocks are fused to their original positions. The final estimate $Q(i)$ is obtained by weighted average, where the weighted weight depends on the coefficient and noise intensity of Wiener filter.

$$
Q(i)=\frac{\sum_{\mathrm{P}} \sum_{\mathrm{Q} \in \mathrm{B}_{P}} W_{P} R_{P Q}}{\sum_{\mathrm{P}} \sum_{\mathrm{Q} \in \mathrm{B}_{P}} W_{P} X_{Q}}, i \in I
$$




\subsection{BM3D Algorithm with an Improved Similarity Measure}

The block matching phase of the BM3D algorithm aims to find image blocks that have the most similar signal components to the reference block. In the assumption of additive Gaussian noise, these blocks coincide with the reference block with the smallest Euclidean distance in the data space; in non-additive Gaussian noise, using Euclidean distance to find similar blocks is no longer optimal. But it can follow Probability principle, based on the actual noise distribution to find a new similarity measure (Parrilli et al., 2011). The BM3D algorithm only selects the Euclidean distance to find the similarity of image blocks, and the detailed information of the image will be lost after filtering. To more accurately match similar image blocks, the Pearson correlation coefficient is introduced to re-determine the similarity between the reference block and the matching block.

$$
\rho=\frac{\operatorname{cov}(P, Q)}{\sqrt{\delta_{P}} \sqrt{\delta_{Q}}}
$$

where $\operatorname{cov}(P, Q)=$ the covariance of the reference block $P$ and the matching block $Q$

$$
\delta_{P}=\text { the variances of the image block } P
$$$$
\delta_{Q}=\text { the variances of the image block } Q
$$

The Pearson correlation coefficient is a statistical index reflecting the degree of similarity between variables, and the value range is $|\rho| \leq 1$. The larger the absolute value of $\rho$, indicating that the similarity between the two images is higher; otherwise, when the value of $\rho$ is 0 , the similarity between the two images is the lowest. When using Euclidean distance to find similar blocks, the smaller $d(P, Q)$, the higher the similarity between image blocks. To be consistent with the Euclidean distance, the definition coefficient is redefined as:

$$
\rho_{\text {final }}=1-\rho^{2}
$$

An improved Euclidean distance calculation method is obtained by combining the determination coefficient. The equation (10) is substituted for $d(P, Q)$ in equation (3):

$$
d_{\text {final }}(P, Q)=d(P, Q) \cdot \rho_{\text {final }}
$$

The specific flow of the algorithm in this paper is as follows:

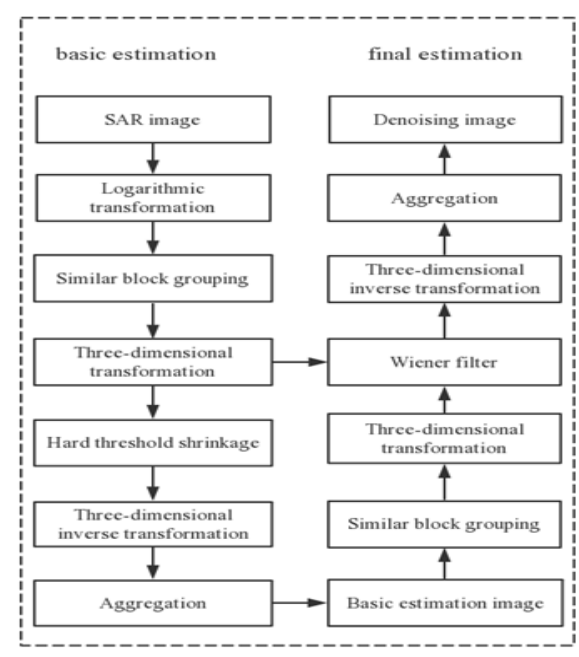

Figure 1. Algorithm flow chart in this paper

\section{EXPERIMENTS AND RESULT ANALYSIS}

To verify the effectiveness of the algorithm in this paper, we selected Sentinel-1A data in parts of Yongcheng county, Henan province. The algorithm in this paper is used to denoise three real SAR images, and the results were compared and analysed with the results of Frost filtering, Kuan filtering, wavelet soft threshold, and SAR-BM3D algorithm. Among them, Frost filtering and Kuan filtering both use a $3 \times 3$ sliding window. Wavelet soft threshold is decomposed and reconstructed by a two-dimensional wavelet. The SAR-BM3D and the algorithm in this paper set the same parameters. The acquisition dates of the three real SAR images selected in this paper are June 3, 2019. These three images include three different scenarios of lakes, urban areas and farmlands as shown in Figure 2. The filtered SAR images are shown in Figure 3.

In this paper, we subjectively evaluate the denoising results of SAR images and use the objective evaluation indexes of mean, variance(var), an equivalent number of looks (ENL) and edge keeping index (EPI) to evaluate. Table 1 shows the objective evaluation indexes of the three SAR images after filtering.

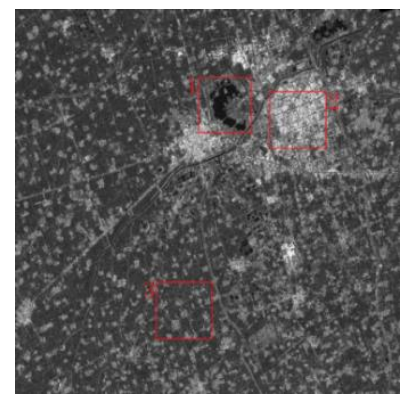

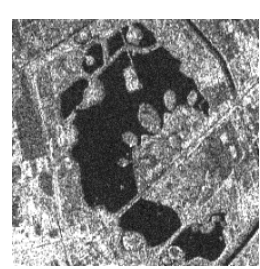

(b)SAR1 (a) Real SAR image

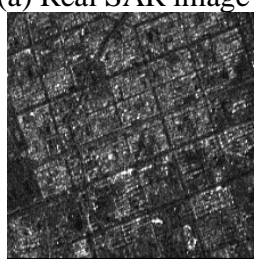

(c)SAR2

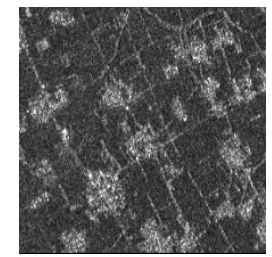

(d)SAR3
Figure 2. Three original SAR images

(where SAR1 contains lakes; SAR2 contains urban areas; SAR3 contains farmlands) 


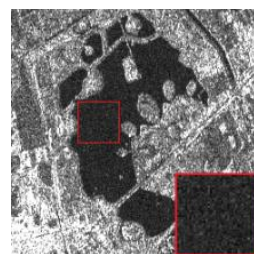

(1-a) Original

image SAR 1

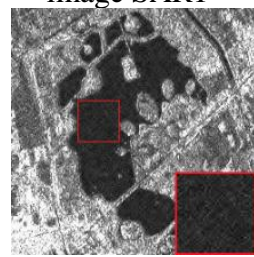

(1-d) Wavelet soft threshold

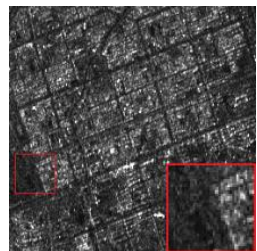

(2-a) Original image SAR2

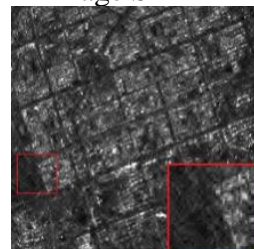

(2-d) Wavelet soft threshold

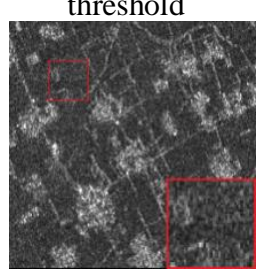

(3-a) Original image SAR3

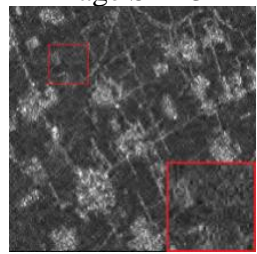

(3-d) Wavelet soft threshold

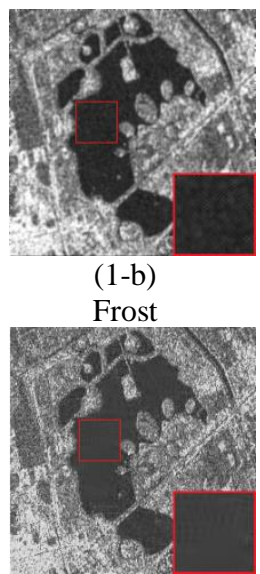

(1-e)

SAR-BM3D

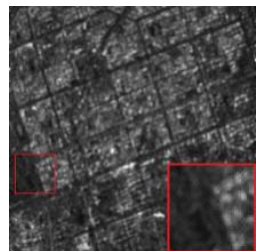

(2-b)

Frost

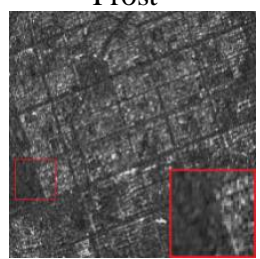

(2-e)

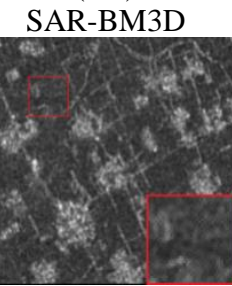

(3-b)

Frost

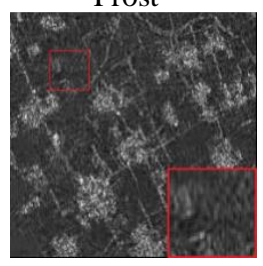

(3-e) SAR-BM3D

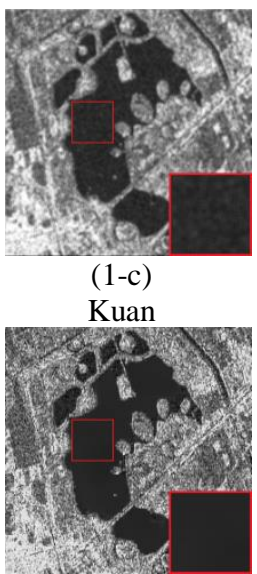

(1-f) Algorithm in this paper

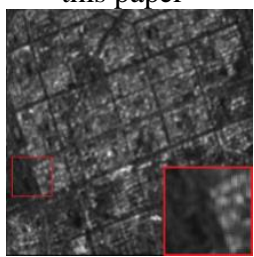

$(2-c)$

Kuan

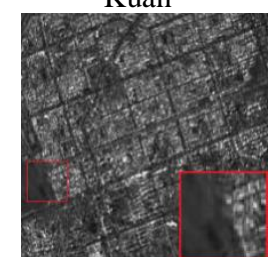

(2-f) Algorithm in this paper

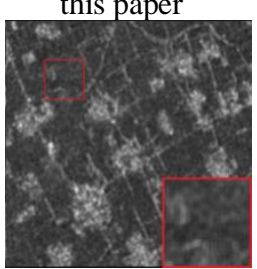

(3-c)

Kuan

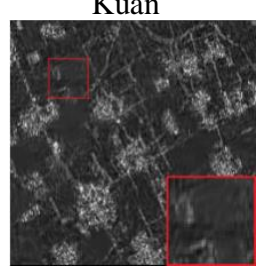

(3-f) Algorithm in this paper
Figure 3. Three SAR images and filtered images

\begin{tabular}{ccccc}
\hline & mean & var & ENL & EPI \\
\hline$(1-a)$ & 122.3467 & 4632.9781 & 3.2309 & 1 \\
$(1-b)$ & 122.3422 & 3355.0922 & 4.4612 & 0.4874 \\
$(1-c)$ & 121.4674 & 3184.0091 & 4.6339 & 0.3495 \\
$(1-d)$ & 122.3247 & 4254.0071 & 3.5175 & 0.8706 \\
$(1-e)$ & 122.1024 & 3728.4477 & 3.9987 & 0.7610 \\
$(1-f)$ & 122.1746 & 2608.5053 & 5.7223 & 0.9098 \\
\hline
\end{tabular}

\begin{tabular}{ccccc}
\hline (2-a) & 70.0356 & 1733.0670 & 2.8302 & 1 \\
(2-b) & 69.9794 & 883.7277 & 5.5414 & 0.3628 \\
(2-c) & 69.7288 & 778.7468 & 6.2435 & 0.2749 \\
(2-d) & 70.0310 & 1420.7735 & 3.4519 & 0.8854 \\
(2-e) & 69.7318 & 975.8139 & 4.9830 & 0.5889 \\
(2-f) & 70.0081 & 690.6800 & 7.0961 & 0.8510 \\
\hline (3-a) & 78.3837 & 1405.2526 & 4.3722 & 1 \\
(3-b) & 78.3068 & 807.8096 & 7.5908 & 0.4647 \\
(3-c) & 78.2108 & 730.8980 & 8.3691 & 0.3160 \\
(3-d) & 78.3808 & 1170.0420 & 5.2507 & 0.8757 \\
(3-e) & 78.8170 & 1090.2384 & 5.6879 & 0.6824 \\
(3-f) & 78.6251 & 691.8806 & 8.9349 & 0.9476 \\
\hline
\end{tabular}

Table 1. Comparison of performance for SAR images denoising

Combined with the SAR images of three types of different scenes and their filtered images. The speckle reduction effect is better after Frost filtering and Kuan filtering, but the image is blurred and a lot of edge detail information is lost. Wavelet soft threshold can be better-maintained image detail information, but the denoising effect is weak and many speckle noises are still distributed in the image. SAR-BM3D algorithm has better denoising effect than Frost filtering and Kuan filtering, but the image edge and texture information retention effect is lower than the wavelet soft-threshold. The algorithm in this paper has the best speckle reduction effect and image edge details to maintain the visual effect.

From the data in Table 1, the mean values of the five filtered results vary slightly compared with original SAR images, indicating that these five filter methods meet the requirements of the mean evaluation index. The ENL data of images after Frost filtering and Kuan filtering are both larger, but EPI data are smaller. Wavelet soft threshold and SAR-BM3D algorithm have larger ENL but smaller EPI. The mean value of the algorithm in this paper is not the closest to the original image, but the variance is the smallest. The ENL and the EPI of the algorithm in this paper are both larger, indicating that the method is superior to other filter algorithms. Comprehensive analysis shows that the algorithm in this paper has good denoising performance, while can effectively suppress coherent speckle noise and maintains image edge detail information well.

\section{CONCLUSION}

This paper uses the Pearson correlation coefficient to improve the Euclidean distance of the BM3D algorithm to find the most similar image blocks. The algorithm in this paper is compared with Frost filtering, Kuan filtering, wavelet soft threshold and SAR-BM3D algorithm in experiments. Subjective visual evaluation and four objective evaluation indexes are used to evaluate the denoised images. The experimental results show that the method can better suppress speckle noise and preserve edge details, and provide higher quality image data for subsequent SAR image processing and interpretation. BM3D algorithm is used to denoise SAR images. Especially on the single-look SAR images, the multiplicative noise of SAR images can be converted into additive noise by logarithmic transformation. However, the transformed noise does not strictly obey the Gaussian distribution, and some errors affect the denoising performance of the algorithm. Therefore, the application of BM3D algorithm to SAR image denoising needs further research. 


\section{ACKNOWLEDGEMENTS}

This paper was support by 2016 National Key Research and Development Plan (2016YFC0803103) of China.

\section{REFERENCES}

Arsenault, H. H., April, G., 1976. Properties of speckle integrated with a finite aperture and logarithmically transformed. Journal of the Optical Society of America, 66(11), 1160-1163.

Buades, A., Coll, B., Morel, J. M., 2005. A non-local algorithm for image denoising. 2005 IEEE Computer Society Conference on Computer Vision and Pattern Recognition (CVPR'05), IEEE, 2005, 2: 60-65.

Chen, S. Y., Li, X. J., 2017. SAR image despeckling based on adaptive non-local means. Systems Engineering and Electronics, 39(12), 2683-2690.

Chierchia, G., EI Gheche, M., Scarpa, G., Verdoliva, L., 2017. Multitemporal SAR image despeckling based on block-matching and collaborative filtering. IEEE Transactions on Geoscience and Remote Sensing, 55(10), 5467-5480.

Cozzolino, D., Parrilli, S., Scarpa, G., Poggi, G., Verdoliva, L., 2014. Fast adaptive nonlocal SAR despeckling. IEEE Geoscience and Remote Sensing Letters, 11(2), 524-528.

Dabov, K., Foi, A., Katkovnik, V., Egiazarian, K., 2007. Image denoising by sparse 3-d transform-domain collaborative filtering. IEEE Transactions on Image Processing, 16(8), 2080-2095.

Li, Y. Z., Shen, T., 2016. SAR image denoising using Fourthorder PDE based on NSWT. Remote Sensing Information, 31(6), 95-99.

Muralimohanbabu, Y., Subramanyam, M. V., Giriprasad, M. N., 2015. A modified bm3d algorithm for SAR image despeckling. Procedia Computer Science, 70(Complete), 69-75.

Parrilli, S., Poderico, M., Angelino, C. V., Verdoliva, L., 2011. A nonlocal SAR image denoising algorithm based on LLMMSE wavelet shrinkage. IEEE Transactions on Geoscience and Remote Sensing, 50(2), 606-616.

Shi, J., Wang, Y., Huang, H. F., Yu, A. X., Li, W., 2016. Application of BM3D algorithm in ocean SAR image denoising. Radar Science and Technology, 14(01), 24-32.

Wang, C. Y., Hu, Z. K., Wu, S. X., 2017. Shearlet domain SAR image denoising method based on Bayesian model. Systems Engineering and Electronics, 39(6), 1250-1255.

Xu, X., Liao, M. S., Bu, F. L., 2000. Speckle filtering method of SAR image based on relative standard deviation. Journal of Remote Sensing, 4(3), 214-218.

Zhao, H. C., Zhou, X. H., Peng, C., Liu. Y. X., Zhang, J. F., Chen, Y. L., 2019. An integrated BM3D method for removing mixed noise in remote sensing image. Geomatics and Information Science of Wuhan University, 44(6), 925-932. 